\title{
Contaminación del medio ambiente y su repercusión en la salud de niños escolares
}

\author{
Guerrero-López Ma. Dolores*, Mendoza-Luna Elizabeth*, Gallegos-Torres Ruth Magdalena**, \\ Xeque-Morales Ángel Salvador***, Palomé-Vega Gabriela***, García-Aldeco Alejandra****
}

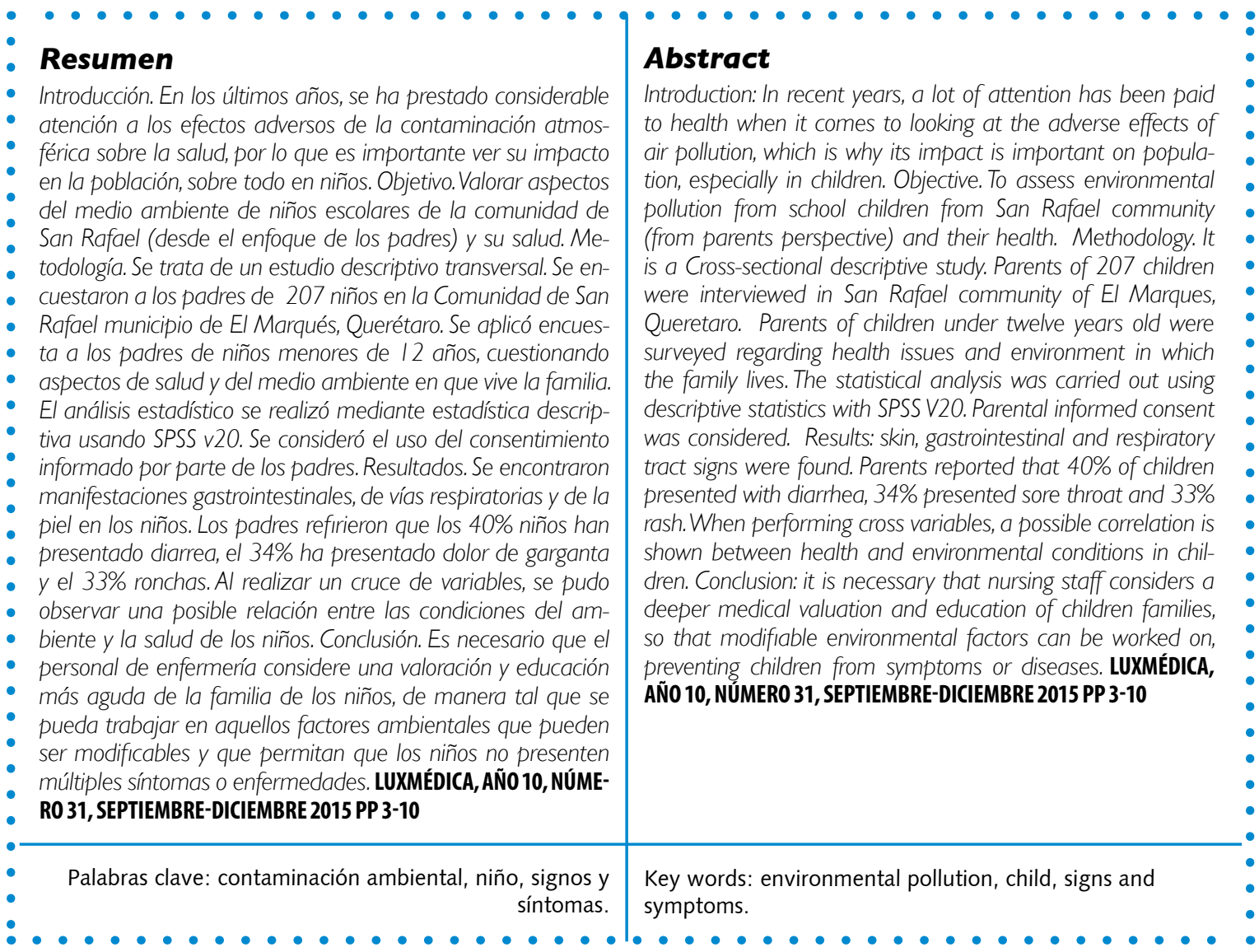

\footnotetext{
* Estudiante Pasante de la Licenciatura en Enfermería, Universidad Autónoma de Querétaro

** Doctora en Ciencias de la Salud, Docente de Tiempo Completo, Facultad de Enfermería, Universidad Autónoma de Querétaro.

*** Maestro en Ciencias, Docente de Tiempo Completo, Facultad de Enfermería, Universidad Autónoma de Querétaro.

**** Maestra en Ciencias de Enfermería, Docente de Tiempo Completo, Facultad de Enfermería, Universidad Autónoma de Querétaro.

***** Maestra en el Desarrollo Infantil, docente de Tiempo Libre, Facultad de Enfermería, Universidad Autónoma de Querétaro.

Fecha de recibido: 10 de julio 2015

Fecha de aceptación: 27 de octubre 2015

Correspondencia: Dra en C Ruth Magdalena Gallegos Torres. Facultad de Enfermería. Universidad Autónoma de Querétaro. Cerro de las Campanas S/N Las Campanas 76010 Santiago de Querétaro, México. Teléfono 01442192 1200. Correo electrónico isismgx@gmail.com
} 


\section{Introducción}

En los últimos años, se ha prestado considerable atención a los efectos adversos de la contaminación atmosférica sobre la salud ${ }^{1}$. La incidencia de varias enfermedades y trastornos pediátricos importantes como asma o trastornos neuronales, está aumentando en varias partes del mundo. Aunque probablemente intervienen diversos factores, algunos pueden deberse a la calidad del medio ambiente en que viven, crecen y juegan los niños ${ }^{2}$.

La relación del medio ambiente en la salud infantil, va a determinar el crecimiento y desarrollo, la salud física y mental, e incluso, el aprendizaje escolar ${ }^{3}$. Según Saldaña ${ }^{4}$, los problemas de salud relacionados con el deterioro del medio ambiente son diversos, entre los que se encuentra el cáncer, primera causa de muerte en los países desarrollados y segunda en los países en vías de desarrollo.

Mezquía ${ }^{5}$ afirman que "los niños son sumamente vulnerables a los peligros ambientales porque respiran más aire, consumen más agua y comida, en relación con su peso, que los adultos. Su sistema inmunitario, su aparato digestivo, su aparato reproductor y su sistema nervioso central son más vulnerables que los de los adultos. Los riesgos ambientales para la salud infantil incluyen un amplio espectro de peligros de distinta naturaleza, en diferentes medios (el agua, el aire, los alimentos y el suelo), en diferentes entornos de exposición, como el hogar, la escuela o la comunidad".

Se considera que los riesgos ambientales inciden en determinadas enfermedades y traumatismos; las principales enfermedades que se presentan en la población infantil son: diarrea, infecciones de las vías respiratorias inferiores, distintos tipos de lesiones accidentales y el paludismo, los cuales cobran cada año la vida de más de cuatro millones de niños, principalmente en los países en vías de desarrollo ${ }^{6}$. La Ciudad de México es una de las ciudades más contaminadas en Latinoamérica. Como resultado de este deterioro ambiental los seres humanos se han visto afectados por la mortalidad prematura, debido a exposiciones al aire contaminado, efectos crónicos tales como la reducción en la condición física, daño permanente a los pulmones y enfisemas, entre otros ${ }^{7}$.

Al respecto, es parte importante del personal de enfermería involucrarse en los fenómenos del medio ambiente que pueden afectar directamente la salud de la población, especialmente de los niños, ya que mucha de la atención se puede llevar a cabo mediante la promoción de la misma, a través de la educación de la población hacia mejores prácticas de cuidado e higiene del medio ambiente que pueda prevenir que los niños enfermen por esta causa. Es por ello que, tomando en consideración que todos los estados de la República cuentan con comunidades con altos niveles de contaminación, se consideró para esta investigación la comunidad de San Rafael, en el Marqués, ya que en la ciudad de Querétaro, es una población que tiene mayor posibilidad de problemas de salud por contaminación de su medio ambiente. 
Por todo lo que se ha señalado es que se busca que en la población de Querétaro se examinen cuáles son los efectos que tienen medios insalubres provocados por la alteración del medio ambiente, en relación a la salud de los escolares y las enfermedades que presentan, por lo tanto el objetivo de esta investigación fue valorar aspectos del medio ambiente en el que vivían niños escolares de la comunidad de San Rafael (desde el enfoque de los padres) y la salud de dichos niños.

| | | | | | | | | | | | | | | | | | | | | | | | | | | | | | | | | | | | | | | | | | | | | | | | | | | | | | | | | | | | | | | | | | | | | | | | | | | | | | | | | | | | | | | | | | | | | | | | | | | | | | | | ||

\section{Material y métodos}

Se trató de un estudio transversal descriptivo, llevado a cabo en la localidad de El Marqués, Querétaro, donde el universo es de 924 menores de edad, por lo que se integra una muestra de 207 niños, esto con base en la fórmula para poblaciones finitas, utilizando un nivel de confianza del $95 \%$.

Se seleccionaron a los niños y niñas de 6 a 12 años cumplidos, bajo el consentimiento de sus padres o tutores para participar en el estudio. Se excluyeron a los niños y niñas con enfermedades agudas; se eliminaron los instrumentos en los que no se obtuvo el $90 \%$ de las respuestas o en los que el padre no diera el consentimiento.

El tipo de muestreo fue por conveniencia; para poder acceder a los niños, se visitó en diversas ocasiones a la comunidad, casa por casa. El instrumento de recolección de datos fue elaborado ex profeso para esta investigación y fue denominado "Aspectos ambientales y salud en niños escolares" el cual cuenta con cuatro apartados: I y II son datos socio-demográficos de los niños y de los padres, el III se refiere a enfermedades infecciosas en los niños, el IV a los contaminantes ambientales. Dicho instrumento se validó con las recomendaciones de Barraza ${ }^{8}$. Las preguntas del instrumento contaban con respuestas múltiples para los posibles síntomas de padecimientos que pudiesen tener los niños con respecto a vías respiratorias, aparato digestivo y piel; fueron dicotómicas para los aspectos de contaminación del medio ambiente.

El instrumento fue piloteado en 20 padres de niños de una comunidad similar a la que se utilizó para la recolección definitiva de datos, trabajando correcciones mínimas de formato más no de contenido. El acercamiento con la comunidad de El Marqués se llevó a cabo de agosto a diciembre del 2014.

Para el procesamiento de los datos de la recolección definitiva, se realizó una base de datos en el programa SPSS v.20. Para el análisis se utilizó estadística descriptiva mediante el uso de frecuencias.

Dentro de los aspectos éticos, se siguieron los principios establecidos en el Reglamento de la Ley General de Salud en Materia de investigación para la Salud 1986, reforma de salud 2012.

| | | | | | | | | | | | | | | | | | | | | | | | | | | | | | | | | | | | | | | | | | | | | | | | | | | | | | | | | | | | | | | | | | | | | | | | | | | | | | | | | | | | | | | | | | | | | | | | | | | | | | | | | | | | | | |

\section{Resultados}

Se encuestaron a los padres de 207 niños en la comunidad. Para caracterizar a los padres encuestados y como un hecho de que dichas características pueden ser un aspecto relevante para la futura educación de estos en materia de medio ambiente, es que se les preguntaron diversos datos sociodemográficos que se describirán a continuación.

De los padres encuestados, se encontraron edades desde los 21 hasta los 47 
años, con un promedio de 31 años, prevaleciendo el género femenino con un $80 \%$; el $45 \%$ de ellos eran casados, el 35\% vivían en unión libre, el $17 \%$ eran madres solteras, y el $2 \%$ viudas.

Los niños van desde los 6 a 12 años con una mayor frecuencia entre 6-7años, prevaleciendo el género femenino con un $51 \%$.

Con respecto a la salud de los niños, a continuación se muestran los hallazgos encontrados.
En relación a las enfermedades gastrointestinales, los padres de familia refirieron que en los últimos meses, el $40 \%$ de los niños presentaron diarrea y el $4 \%$ ha presentado deshidratación (gráfica 1).

De las enfermedades respiratorias, los padres indicaron que el $34 \%$ de los niños presentó dolor de garganta y el $4 \%$ ninguna enfermedad (gráfica 2).

Con respecto a los síntomas de la piel el $33 \%$ de los niños ha tenido ronchas y el $5 \%$ ardor (gráfica 3).

\section{Gráfica 1 \\ Enfermedades gastrointestinales que han presentado los niños \\ Diarrea Vómito Deshidratación Sin apetito $\square$ Ninguna}

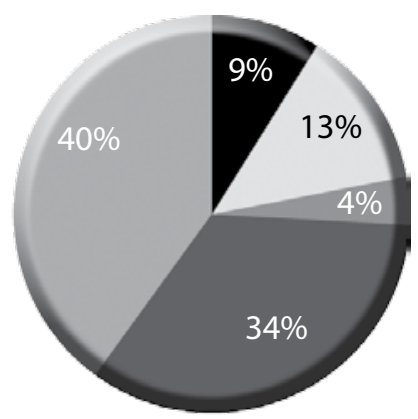

Fuente: encuesta aplicada, 2014.

\section{Gráfica 2}

Enfermedades respiratorias que han presentado los niños

Tos Dolor de garganta Congestión Gripe resfriado Ninguna

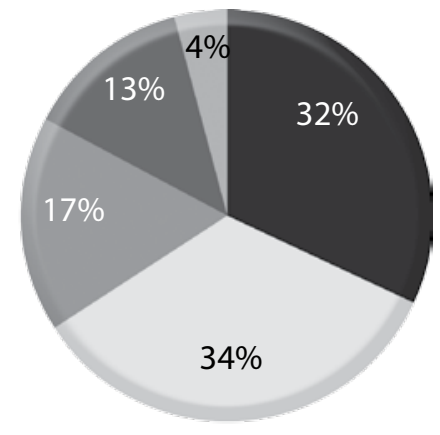

Fuente: encuesta aplicada, 2014. 


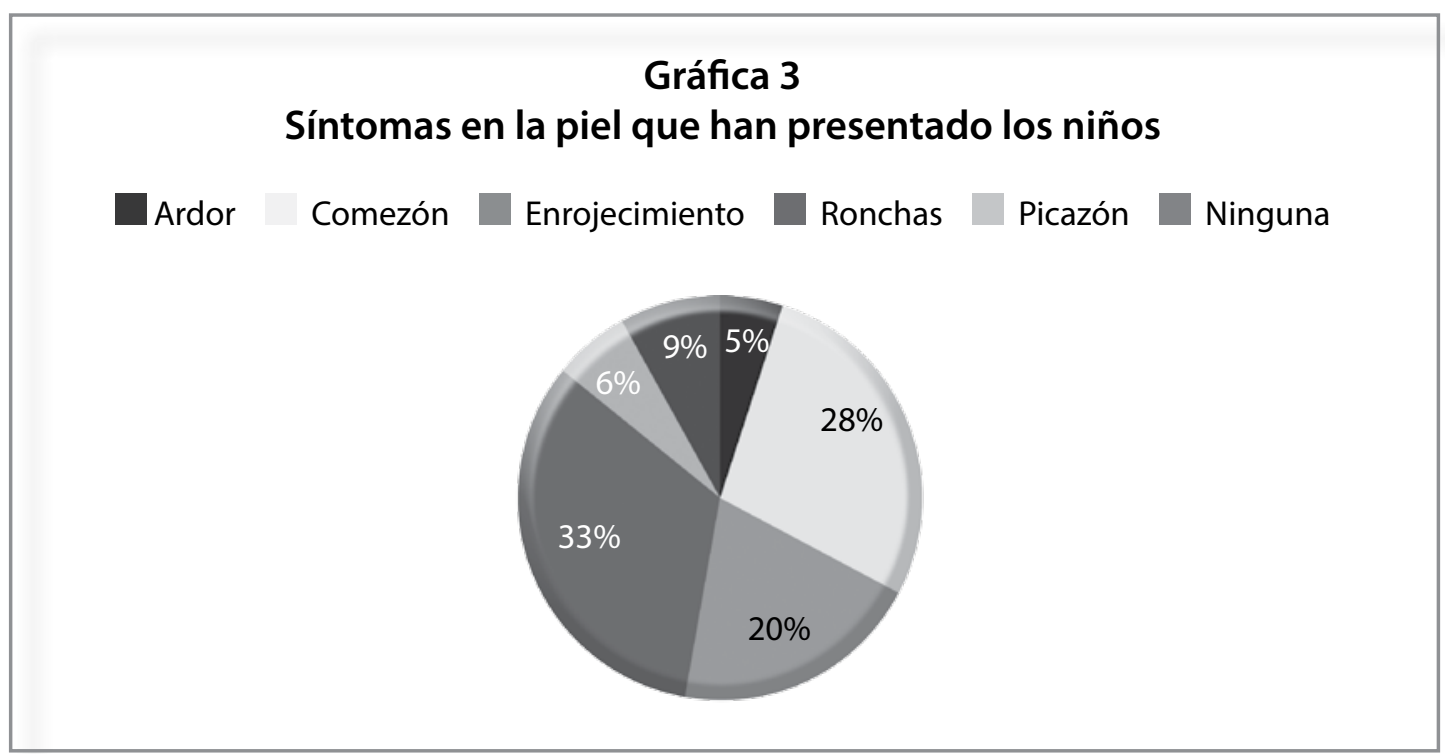

Fuente: encuesta aplicada, 2014.

En relación a los aspectos del medio ambiente, vinculados con la vivienda, el $84 \%$ de los padres mencionaron que el piso de su casa está construido de concreto, el $14 \%$ de loseta y el $2 \%$ de tierra. En el $82 \%$ las casas no están cerca de algún relleno sanitario. El 94\% refirió contar con baño propio con drenaje y el 3\% defeca al aire libre. El 73\% tienen mascotas dentro de su hogar, siendo el perro con $71 \%$ la mascota que más se encuentra, aunque también hay gatos, aves y otro tipo de animales.

El $87 \%$ de las casas se encuentra cerca de un criadero de animales, aunque el $81 \%$ mencionó que no tiene criadero de animales dentro de su casa; de los animales que existen en los criaderos prevalecieron las vacas con un $84 \%$.

De acuerdo con las encuestas, el $67 \%$ de los techos de las casas están construidos de concreto; el $61 \%$ de los padres fuma dentro de su hogar. El $97 \%$ de las familias cocinan con gas y sólo un $3 \%$ con leña, y cuentan con agua potable el $87 \%$ de las familias; el $75 \%$ del agua que se consume es de la llave y el $25 \%$ es embotellada. Un $63 \%$ de las familias cuentan con tinaco. El $68 \%$ de los niños se bañan tres veces por semana.

El $64 \%$ de los padres acostumbra a quemar basura, de los cuales el $63 \%$ refirió que ésta la queman cerca de su casa. El $89 \%$ de los padres consideran que el aire tiene mal olor; relacionado con lo anterior, el $74 \%$ acostumbra a utilizar aerosoles.

Al realizar el cruce de variables, se pudieron observar aspectos interesantes (tabla 1, tabla 2 y tabla 3 ). 


\section{Tabla I}

Condiciones del medio ambiente presentes en niños que han manifestado enfermedades gastrointestinales

\begin{tabular}{|lll|}
\hline Variable & \multicolumn{2}{c|}{ Enfermedad gastrointestinales } \\
& Sí & No \\
\hline Contacto con relleno sanitario & 30 & 152 \\
\hline Baño propio con drenaje & 170 & 12 \\
\hline Defeca al aire libre & 7 & 175 \\
\hline Tiene letrina & 13 & 169 \\
\hline
\end{tabular}

Fuente: encuesta aplicada, 2014.

\section{Tabla 2}

\section{Condiciones del medio ambiente presentes en niños que han manifestado enfermedades respiratorias.}

\begin{tabular}{|lll|}
\hline Variable & Enfermedad respiratorias & \\
& Sí & No \\
\hline Tiene mascotas & 142 & 47 \\
\hline La casa se ubica cerca de un criadero & 168 & 21 \\
\hline Cerca de su hogar existe una fábrica & 36 & 153 \\
\hline Algún integrante de la familia fuma & 124 & 65 \\
\hline Cerca de su hogar acostumbra a quemar basura & 75 & 114 \\
\hline El aire tiene mal olor & 167 & 22 \\
\hline Usa aerosol & 141 & 48 \\
\hline
\end{tabular}

Fuente: encuesta aplicada, 2014.

\section{Tabla 3}

Condiciones del medio ambiente presentes en niños que han manifestado enfermedades de la piel.

\begin{tabular}{|lll|}
\hline Variable & Enfermedad - síntomas de la piel & \\
& Sí & No \\
\hline Tiene mascotas & 128 & 48 \\
\hline La casa se ubica cerca de un criadero & 153 & 23 \\
\hline Cada cuanto se baña. & & \\
\hline 3 veces por semana & 129 & \\
\hline Diario & 47 & \\
\hline
\end{tabular}

Fuente: encuesta aplicada, 2014. 


\section{Discusión}

Es necesario considerar la realización de estudios en donde enfermería se involucre en el conocimiento que la población tiene sobre el medio ambiente, la contaminación y la salud en general, de adultos y sobre todo niños. En este sentido, se reconoce que la principal limitación para la realización de este estudio fue la escasa información que hay sobre salud de niños y contaminación que hay en el ambiente en que se desenvuelven, sin embargo, se reconoce que esta aproximación aporta puntos relevantes para la profesión.

Por ejemplo, en el estudio de Quiroz ${ }^{9}$, realizado a 1,627 niños menores de 10 años, en niños de cinco municipios carboníferos del César, Colombia, se encontró que el $17,0 \%$ de éstos faltaron a clases al menos un día del último mes por presentar enfermedad respiratoria aguda. Relacionado con esto, en esta investigación se encontró que los niños sí han tenido manifestaciones de problemas respiratorios como dolor de garganta (34\%), tos (32\%), congestión (17\%) y gripe-resfriado (13\%), aunque no se cuestionó a los padres si estas situaciones favorecieron que el niño no asistiera a la escuela.

Al tomar en cuenta las enfermedades respiratorias, Zayas ${ }^{10}$ menciona que el $60 \%$ de las enfermedades asociadas a infecciones respiratorias están vinculadas con la exposición a la contaminación ambiental. Estas exposiciones se han vinculado con la ocurrencia del asma, bronquitis, neumonía e infecciones del oído en niños, lo cual coincide con el estudio realizado donde el $60 \%$ de los niños padece de enfermedades respiratorias y algún integrante de la familia fuma.

Por otra parte, con respecto a las enfermedades gastrointestinales, Klanian, Duran, Isla, Suárez y Alarcón ${ }^{11}$, mencionan que el $32.1 \%$ de los residentes de la comunidad de San Simón padecieron de enfermedad diarreica, lo cual está relacionado con los resultados encontrados en esta investigación donde se presentó la diarrea en un $40 \%$ de los niños.

Tras el análisis de datos de este estudio, se encontró que $7.7 \%$ de los niños ha presentado síntomas en la piel como ronchas, comezón y enrojecimiento, encontrando que en sus casas hay mascotas y la casa está ubicada cerca de un criadero; esto coincide con lo que menciona Bedolla, Barrera, Morales $^{12}$ tras su estudio en niños escolares (de 6 a 12 años) de Ciudad Guzmán, México; trabajaron con un total de 740 niños, donde la prevalencia de dermatitis atópica fue de $3.0 \%$, encontrando también relación con Mero ${ }^{13}$, ya que realizó un estudio elaborado a niños escolares de 5 a 10 años, en la consulta externa del hospital de San Rafael Rodríguez Zambrano, donde la dermatitis atópica está afectando actualmente a más de del 10\% de los niños, debido a la contaminación ambiental. 


\section{Conclusiones}

Es claro el hecho de que muchos de los problemas de salud son generados por factores vinculados con el medio ambiente, como el cambio climático o contaminación atmosférica, sin embargo, al respecto, es evidente que existen desigualdades entre países desarrollados y en vías de desarrollo, donde los que se ven más afectados por la contaminación ambiental son los niños.

Existe una estrecha relación ente la salud de los niños y el medio ambiente donde se desenvuelven, ya que el ambiente establece condiciones que impactan en la salud, manifestando distintas enfermedades que limitan la calidad de vida. En este sentido, es importante reconocer aquellos contaminantes que se consideran como determinantes principales para presentar condiciones insalubres que se manifiestan con enfermedades, principalmente con afecciones respiratorias, gastrointestinales y dermatológicas.

De esta manera, este tipo de información debe ser considerada por el personal de enfermería, sobre todo aquel que trabaja en el primer nivel de atención, de manera tal que se puedan atender factores (a través de la educación u orientación) que sí pueden ser modificables por la familia de los niños, como por ejemplo el baño.

Para poder reforzar los resultados como los arrojados por esta investigación, es necesario llevar a cabo estudios de correlación y posteriormente de casos y controles con el uso de un grupo control, o bien el abordaje cualitativo que permita identificar la percepción de la familia sobre la contaminación del medio ambiente.

\section{Bibliografía}

1. Rodríguez Villamizar LA, Herrera López $A B$, Castro Ortiz H, Niederbacher Velázquez J, Vera Cala LM. Incidencia de síntomas respiratorios y su asociación con contaminación atmosférica en preescolares: un análisis multinivel. Cad Saúde Pública, Rio Janeiro. 2010;26(7):1411-8.

2. París $M E$, Bettini $M$, Molina $H$, Mieres JJ, Bravo $V$, Ríos JC. La importancia de la salud ambiental y el alcance de las unidades de pediatría ambiental. Rev Med Chil. 2009;137(1):101-5.

3. Hernández Celis TC. Implementación del programa hospital verde en el instituto del corazón de bucaramanga. 2010.

4. Saldaña García L, Aguilar Batista L, García Triana BE, Saldaña Bernabeu CA. Sociedad, Contaminación ambiental y carcinogénesis química. Memorias Conv Int Salud Pública Cuba Salud. 2012.

5. Mezquía Valera A, Cumba Abreu C, Aguilar Valdez J, García Roche R, Acosta Quintana L. Condiciones ambientales riesgosas para las infecciones respiratorias agudas en escolares de primaria. Rev Cuba Med Gen Integr. 2011;27(2): 155-160

6. Prüss Üstün A, Corvalan C. Ambientes saludables y prevención de enfermedades: hacia una estimación de la carga de morbilidad atribuible al medio ambiente. Ambientes saludables y prevención de enfermedades. Organización Mundial de la Salud. 2006.
7. Escobedo F, Chacalo A. Estimación preliminar de la descontaminación atmosférica por el arbolado urbano de la ciudad de México. Rev Cienc y Tec América. 2008;33(1):29-33.

8. Barraza MA. La consulta a expertos como estrategia para la recolección de evidencias de validez basadas en el contenido. 2007.

9. Quiroz Arcentales L. Enfermedad y síntomas respiratorios en niños de cinco municipios carboníferos del Cesar, Colombia. Rev Salud Pública. 2013;15(1):6679.

10. Zayas Mujica R, Ulises CC. Los tóxicos ambientales y su impacto en la salud de los niños. Rev Cubana Pediatr. 2007;79(2).

11. Klanian MG, Durán Casanova JG, Isla Esquivel ML, Suárez Wegan E, Alarcón Sánchez A. Estudio de factores predisponentes de enfermedad diarreica aguda en la comunidad de San Simón, Yucatán en base a un análisis de vulnerabilidad nutricional y ambiental. Rev académica Univ Costa Rica. 2011;9(1).

12. Bedolla Barajas M, Cuevas Ríos G, García Barboza E, Barrera Zepeda AT, Morales Romero J. Prevalencia y factores asociados a la rinitis alérgica en escolares de ciudad Guzmán, México. Rev Investig Clínica. 2010;62(3):244-51.

13. Mero Chávez TA, Mero López JT. Dermatitis atópica en escolares de 5 a 10 años, estudio de casos en la consulta externa del hospital Rafael Rodriguez Zambrano de mayo del 2012 a marzo del 2013. 